2006

\title{
Monodomain Dynamics for Rigid Rod and Platelet Suspensions in Strongly Coupled Coplanar Linear Flow and Magnetic Fields. II. Kinetic theory
}

\author{
M. Gregory Forest
}

Sarthok Sircar

Qi Wang

Ruhai Zhou

Old Dominion University, rzhou@odu.edu

Follow this and additional works at: https://digitalcommons.odu.edu/mathstat_fac_pubs

Part of the Engineering Physics Commons, Fluid Dynamics Commons, and the Mathematics Commons

\section{Repository Citation}

Forest, M. Gregory; Sircar, Sarthok; Wang, Qi; and Zhou, Ruhai, "Monodomain Dynamics for Rigid Rod and Platelet Suspensions in Strongly Coupled Coplanar Linear Flow and Magnetic Fields. II. Kinetic theory" (2006). Mathematics \& Statistics Faculty Publications. 14.

https://digitalcommons.odu.edu/mathstat_fac_pubs/14

\section{Original Publication Citation}

Forest, M. G., Sircar, S., Wang, Q. \& Zhou, R. (2006). Monodomain dynamics for rigid rod and platelet suspensions in strongly coupled coplanar linear flow and magnetic fields. II. Kinetic theory. Physics of Fluids, 18(10), 103102 doi:10.1063/1.2359232 


\title{
Monodomain dynamics for rigid rod and platelet suspensions in strongly coupled coplanar linear flow and magnetic fields. II. Kinetic theory
}

\author{
M. Gregory Forest \\ Departments of Mathematics and Biomedical Engineering, University of North Carolina, \\ Chapel Hill, North Carolina 27599-3250 \\ Sarthok Sircar \\ Department of Mathematics, Florida State University, Tallahassee, Florida 32306 \\ Qi Wang ${ }^{\text {a) }}$ \\ Department of Mathematics, Florida State University, Tallahassee, Florida 32306 \\ and School of Mathematics, Nankai University, Tianjin 300071, People's Republic of China \\ Ruhai Zhou \\ Department of Mathematics and Statistics, Old Dominion University, Norfolk, Virginia 23529
}

(Received 28 March 2006; accepted 7 September 2006; published online 12 October 2006)

\begin{abstract}
We establish reciprocity relations of the Doi-Hess kinetic theory for rigid rod macromolecular suspensions governed by the strong coupling among an excluded volume potential, linear flow, and a magnetic field. The relation provides a reduction of the flow and field driven Smoluchowski equation: from five parameters for coplanar linear flows and magnetic field, to two field parameters. The reduced model distinguishes flows with a rotational component, which map to simple shear (with rate parameter) subject to a transverse magnetic field (with strength parameter), and irrotational flows, for which the reduced model consists of a triaxial extensional flow (with two extensional rate parameters). We solve the Smoluchowski equation of the reduced model to explore: (i) the effect of introducing a coplanar magnetic field on each sheared monodomain attractor of the Doi-Hess kinetic theory and (ii) the coupling of coplanar extensional flow and magnetic fields. For (i), we show each sheared attractor (steady and unsteady, with peak axis in and out of the shearing plane, periodic and chaotic orbits) undergoes its own transition sequence versus magnetic field strength. Nonetheless, robust predictions emerge: out-of-plane degrees of freedom are arrested with increasing field strength, and a unique flow-aligning or tumbling/wagging limit cycle emerges above a threshold magnetic field strength or modified geometry parameter value. For (ii), irrotational flows coupled with a coplanar magnetic field yield only steady states. We characterize all (generically biaxial) equilibria in terms of an explicit Boltzmann distribution, providing a natural generalization of analytical results on pure nematic equilibria [P. Constantin, I. Kevrekidis, and E. S. Titi, Arch. Rat. Mech. Anal. 174, 365 (2004); P. Constantin, I. Kevrekidis, and E. S. Titi, Discrete and Continuous Dynamical Systems 11, 101 (2004); P. Constantin and J. Vukadinovic, Nonlinearity 18, 441 (2005); H. Liu, H. Zhang, and P. Zhang, Comm. Math. Sci. 3, 201 (2005); C. Luo, H. Zhang, and P. Zhang, Nonlinearity 18, 379 (2005); I. Fatkullin and V. Slastikov, Nonlinearity 18, 2565 (2005); H. Zhou, H. Wang, Q. Wang, and M. G. Forest, Nonlinearity 18, 2815 (2005)] and extensional flow-induced equilibria [Q. Wang, S. Sircar, and H. Zhou, Comm. Math. Sci. 4, 605 (2005)]. We predict large parameter regions of bi-stable equilibria; the lowest energy state always has principal axis aligned in the flow plane, while another minimum energy state often exists, with primary alignment transverse to the coplanar field. (C) 2006 American Institute of Physics. [DOI: $10.1063 / 1.2359232]$
\end{abstract}

\section{INTRODUCTION}

Imposed magnetic fields typically induce magnetic moments on rod and platelet particles, even when the particles do not have permanent dipoles. This effect is an appealing candidate to control the orientational distribution of the particle ensemble, especially during shear-dominated flows of nematic liquid crystal polymers and anisotropic colloidal dispersions. The present paper will shed light on this question

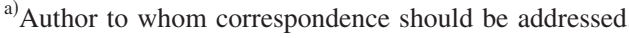

of the coupling between coplanar linear flows and magnetic fields. However, to make these predictions, we first take a detour to develop the models that will be implemented in our numerical and analytical approach.

The extended Doi-Hess kinetic theory for macromolecular rigid rods and platelets in a viscous solvent, governed by excluded volume interactions, an imposed linear planar flow, and an imposed coplanar magnetic field, involves many parameters (detailed below). Classical symmetries can be developed and extended to significantly reduce the number of parameters, and thus explore the original experimental pa- 
rameter space. We begin with an explanation of this theoretical "preconditioning" of the problem.

A classical analogy has been utilized in the (small molecule) liquid crystal literature, wherein a magnetic field is equivalent to uniaxial extensional flow. ${ }^{1}$ This analogy allows one experiment to be replaced by another more convenient one; yet the trade-off is "one for one" experimentally, which translates to a 1:1 map between solutions of the corresponding model equations. Such correspondences are often called reciprocity relations. For liquid crystal polymers, or macromolecular rod suspensions, it is common knowledge that either an extensional flow or magnetic field will align the rod ensemble, and the orientational distribution becomes more peaked along the field axis with increased strength. This experimental phenomenon is borne out by an extension of the above classical analogy from liquid crystal theory to the DoiHess kinetic theory. Recently, the authors ${ }^{2}$ observed this straightforward extension and established a more substantial, qualitatively different correspondence, which results from the coupling of nonlocal excluded volume interactions of rods and the Jeffery orbit of a single rigid rod (or platelet). Namely, all linear planar flows of a given rod suspension can be reduced either to a simple shear or to a simple extensional flow, each with a modified particle shape parameter. In effect, one flow parameter can be removed by an orthogonal change of coordinates, and a second flow parameter can be absorbed into a modified particle shape. The reciprocity relation affords a two-parameter reduction, which was implemented to study shear-extensional flow response diagrams of nematic polymers. ${ }^{2}$ In particular, the reduced model easily establishes the requisite extension rate required to tame complex shear-dominated limit cycles and chaotic dynamics.

The phenomena of interest in the present paper surround coupling a coplanar magnetic field to the previous study. The Doi-Hess kinetic model for magnetic and coplanar linear flows for rigid rod suspensions, with an excluded volume potential of Maier-Saupe chosen here, yields a fiveparameter space of the flow and magnetic field; the model also requires the particle geometry and the strength of the excluded volume potential. Clearly, a model reduction will be valuable for exploring phase response diagrams, and for determining how magnetic fields might tame sheared attractors or modify extensional flow equilibria. That is the focus of this paper.

Bandhar and $\mathrm{Wiest}^{3}$ derived kinetic model equations for the coupling of magnetic and flow fields, extending the DoiHess theory ${ }^{4,5}$ to include both permanent and induced dipole responses to an imposed magnetic field. In this paper, the authors derive a reciprocity principle of the kind described above, relating the solutions of the Smoluchowski equation for two model systems of Doi-Hess type with a common ingredient of a nonlocal excluded volume potential: a fiveparameter family of coplanar linear flow and magnetic fields; and 2 two-parameter families, one of simple shear with a transverse magnetic field along the vorticity axis, a second of a triaxial extensional flow. The reduced shear model with transverse magnetic field allows the solution of all planar rotational flows with coplanar magnetic field, and the extensional flow reduced model allows the solution of any planar irrotational flow with coplanar magnetic field. These observations, coupled with the solution of the reduced (or target) model or the experiment that it models, significantly reduce the cost of experiments and model solution methods.

Our goal here is to solve the solutions of the Smoluchowski equation for each of the two target kinetic models. For the target shear-transverse magnetic field system, we generalize our spherical harmonic expansion algorithm and code $^{6,7}$ to include the magnetic field along the vorticity axis. We note that this extension does not require a complicated rederivation of the spherical harmonic amplitude equations, which would be the case if the reciprocity relation were not recognized nor exploited. Furthermore, the reciprocity principle explicitly shows that there is a three-parameter redundancy in the original coplanar flow-magnetic field system, leading to potentially wasteful simulation time and effort to discover that entire three-parameter families of solutions all collapse onto an identical probability distribution modulo an orthogonal change of coordinates. For the target extensional flow system, we establish analytical results first, which follow immediately from the fact that the extensional flow retains the gradient structure of the Smoluchowski equation with excluded volume potential. Thereby, all steady state probability distribution functions (pdfs) have a Boltzmann distribution, which is explicitly parametrized by the two order parameters of the second moment of the pdf. These analytical results follow several recent advances, initiated by Constantin and collaborators, followed by other research groups. ${ }^{1,8-13}$ We then solve the steady state equations numerically to convey the various stable and unstable equilibria that arise in the coupling between extensional flow and a magnetic field.

\section{MATHEMATICAL FORMULATION}

We briefly review the mathematical formulation of the Doi-Hess kinetic theory for homogeneous flows of rigid spheroids (rods or platelets) immersed in a viscous solvent subject to an imposed magnetic field. ${ }^{5,14-16}$ We allow a general excluded volume potential

$$
V_{i}(\mathbf{m}, \mathbf{x}, t)=\nu k T \int_{\left\|\mathbf{m}^{\prime}\right\|=1} B\left(\mathbf{m}, \mathbf{m}^{\prime}\right) f\left(\mathbf{m}^{\prime}, \mathbf{x}, t\right) d \mathbf{m}^{\prime},
$$

where $\nu$ is the number density of rods or platelets (modeled as spheroids here at large and small aspect ratios, respectively), $\mathbf{m}$ and $\mathbf{m}^{\prime}$ are unit vectors for the axes of symmetry of a given spheroid, $B\left(\mathbf{m}, \mathbf{m}^{\prime}\right)$ is the excluded volume and $f$ is the orientational probability density function of the ensemble of spheroids. In the presence of an imposed magnetic field, an induced magnetic moment develops; we assume intrinsic magnetic moments and their magnetic dipole-dipole interaction are negligible (nondipolar spheroids). For such systems, the potential due to the external field is given by $3,4,17$

$$
V_{H}=-\frac{\chi_{\alpha}}{2}(\mathbf{H} \cdot \mathbf{m})^{2},
$$

where $\mathbf{H}$ is the magnetic field vector, and $\chi_{\alpha}$ (normally positive for paramagnetic materials and negative for diamagnetic 
materials) is the difference between the susceptibility parallel and perpendicular to the spheroid principal axis, known as the magnetic anisotropy.

The rotational transport equation for the orientational pdf is given by the Smoluchowski equation of Doi and Hess: ${ }^{3-5}$

$$
\begin{aligned}
& \frac{d f}{d t}=\mathcal{R} \cdot\left[D_{r}(a) f \mathcal{R}\left(\mu+\frac{1}{k T} V_{H}\right)\right]-\mathcal{R} \cdot[\mathbf{m} \times \dot{\mathbf{m}} f], \\
& \dot{\mathbf{m}}=\mathbf{W} \cdot \mathbf{m}+a[\mathbf{D} \cdot \mathbf{m}-\mathbf{D}: \mathbf{m m m}],
\end{aligned}
$$

where $D_{r}(a)$ is the rotational diffusivity (assumed to be constant in this study), $\mathcal{R}=\mathbf{m} \times(\partial / \partial \mathbf{m})$ is the rotational gradient operator, and $(d / d t)(\bullet)$ denotes the material derivative $(\partial / \partial t)(\cdot)+\mathbf{v} \cdot \nabla(\cdot), \mathbf{D}$, and $\mathbf{W}$ are the rate-of-strain and vorticity tensors, respectively; $a$ is a geometry or shape parameter defined by $a=\left(r^{2}-1\right) /\left(r^{2}+1\right)$ in terms of the spheroidal aspect ratio $r ; \mu=\ln f+(1 / k T) V_{i}$ is the normalized chemical potential, and $V_{i}$ is the intermolecular potential.

By substituting the magnetic field potential into the Smoluchowski equation, we show that the Smoluchowski equation can be rewritten into a form with a modified Jeffery orbit equation for $\mathbf{m}$ containing a transport term due to the magnetic forcing:

$$
\begin{aligned}
& \mathbf{m} \times(\dot{\mathbf{m}}+\chi \mathbf{H H} \cdot \mathbf{m}) \\
&= \mathbf{m} \times\left\{\mathbf{W} \cdot \mathbf{m}+\left[a \mathbf{D}+\chi\left(\mathbf{H H}-\frac{H^{2}}{k_{0}} \mathbf{I}\right)\right]\right. \\
&\left.\cdot \mathbf{m}-\left[a \mathbf{D}+\chi\left(\mathbf{H H}-\frac{H^{2}}{l} \mathbf{I}\right)\right]: \mathbf{m m m}\right\},
\end{aligned}
$$

where $\chi=D_{r} \chi_{\alpha} / k T$ is a normalized magnetic anisotropy, $k_{0}, l$ can be any nonzero numbers, and $H=\|\mathbf{H}\|$ is the magnitude of the magnetic field.

Recall the Smoluchowski equation absent of external fields is invariant under orthogonal transformations, which reflects the orientational degeneracy of ordered equilibria $f_{\text {eq }}$ due to excluded volume interactions. That is, nematic equilibria have a specified Boltzmann distribution function and unique uniaxial order parameter, but the principal axis of orientation is arbitrary. If $\mathbf{n}=\mathbf{U} \cdot \mathbf{m}$, where $\mathbf{U}$ is an arbitrary orthogonal matrix, and the flow field is absent $(\dot{\mathbf{n}}=0)$, then Eq. (3) leads to

$$
\frac{d \tilde{f}}{d t}=\mathcal{R}_{n} \cdot\left[D_{r}(a) \tilde{f} \mathcal{R}_{n} \tilde{\mu}\right]-\mathcal{R}_{n} \cdot[\mathbf{n} \times(\chi \tilde{\mathbf{H}} \tilde{\mathbf{H}} \cdot \mathbf{n}) \tilde{f}]
$$

where the pdf $\tilde{f}=\tilde{f}(\mathbf{n}, \mathbf{x}, t)=f\left(\mathbf{U}^{T} \cdot \mathbf{n}, \mathbf{x}, t\right), \quad \mathcal{R}_{n}=\mathbf{n} \times(\partial / \partial \mathbf{n})$, $\tilde{\mathbf{H}}=\mathbf{U} \cdot \mathbf{H}$ is the rotated external field, and $\tilde{\mu}=\ln \tilde{f}$ $+(1 / k T) V_{i}(\mathbf{n}, \mathbf{x}, t) . \mathbf{U}^{T}$ denotes the transpose of the matrix $\mathbf{U}$.
If we denote $\operatorname{SU}(2, \mathbf{H})=\{\mathbf{U} \mid \mathbf{U} \cdot \mathbf{H}=\mathbf{H}\}$, the rotational group on the plane perpendicular to the field direction $\mathbf{H}$, the Smoluchowski equation absent of flows is invariant under $\mathrm{SU}(2, \mathbf{H})$. Therefore, the reduced degeneracy of the ordered equilibrium persists.

\section{REDUCED (TARGET) MODELS BASED ON RECIPROCITY RELATIONS}

We briefly discuss the reduction from the Smoluchowski equation for coplanar linear flows and magnetic fields to the reduced or target model. Consider a linear planar flow field

$$
\mathbf{v}=\left(v_{11} x+v_{12} y, v_{21} x-v_{11} y, 0\right),
$$

where $v_{i j}$ are constants, with gradient

$$
\nabla \mathbf{v}=\left(\begin{array}{lll}
v_{11} & v_{12} & 0 \\
v_{21} & -v_{11} & 0 \\
0 & 0 & 0
\end{array}\right)
$$

and a coplanar magnetic field $\mathbf{H}=\left(H_{1}, H_{2}, 0\right)^{T}$. Let

$$
p=\frac{1}{2}\left(v_{12}+v_{21}\right), \quad q=\frac{1}{2}\left(v_{12}-v_{21}\right),
$$

where $-2 q$ is the vorticity. The rate-of-strain tensor and the vorticity tensor are given, respectively, by

$$
\begin{aligned}
& \mathbf{W}=\frac{1}{2}\left(\nabla \mathbf{v}-\nabla \mathbf{v}^{T}\right)=\left(\begin{array}{lll}
0 & q & 0 \\
-q & 0 & 0 \\
0 & 0 & 0
\end{array}\right), \\
& \mathbf{D}=\frac{1}{2}\left(\nabla \mathbf{v}+\nabla \mathbf{v}^{T}\right)=\left(\begin{array}{lll}
v_{11} & p & 0 \\
p & -v_{11} & 0 \\
0 & 0 & 0
\end{array}\right) .
\end{aligned}
$$

$q \neq 0$ corresponds to a rotational flow field with a nonvanishing vorticity tensor. By choosing $k_{0}=l=2$ in (4), the upper left $2 \times 2$ submatrix in $\mathbf{W}+a\left[\mathbf{D}+\chi \mathbf{H H}-\left(\chi H^{2} / 2\right)\left(\mathbf{e}_{1} \mathbf{e}_{1}\right.\right.$ $\left.\left.+\mathbf{e}_{2} \mathbf{e}_{2}\right)\right]$ qualifies as an effective rate-of-strain tensor. We rewrite $\mathbf{W}=q \mathbf{W}_{0}$ and

$$
a \mathbf{D}+\chi \mathbf{H H}-\frac{\chi H^{2}}{2}\left(\mathbf{e}_{1} \mathbf{e}_{1}+\mathbf{e}_{2} \mathbf{e}_{2}\right)=\lambda \mathbf{U}^{T} \cdot \mathbf{D}_{0} \cdot \mathbf{U}
$$

where $\mathbf{W}_{0}$ and $\mathbf{D}_{0}$ are normalized vorticity and rate-of-strain tensors, respectively, for the pure shear velocity field $\mathbf{v}=(2 y, 0,0)$, and $\mathbf{U}$ is the rotational matrix, 


$$
\begin{aligned}
& \mathbf{W}_{0}=\left(\begin{array}{lll}
0 & 1 & 0 \\
-1 & 0 & 0 \\
0 & 0 & 0
\end{array}\right), \quad \mathbf{D}_{0}=\left(\begin{array}{lll}
0 & 1 & 0 \\
1 & 0 & 0 \\
0 & 0 & 0
\end{array}\right), \\
& \mathbf{U}=\left(\begin{array}{lll}
\cos \theta & \sin \theta & 0 \\
-\sin \theta & \cos \theta & 0 \\
0 & 0 & 1
\end{array}\right), \\
& \cos 2 \theta=\frac{b}{\sqrt{b^{2}+c^{2}}}, \quad \sin 2 \theta=-\frac{c}{\sqrt{b^{2}+c^{2}}}, \\
& b=a p+\chi H_{1} H_{2}, \quad c=a v_{11}+\chi \frac{H_{1}^{2}-H_{2}^{2}}{2}, \\
& \lambda=\sqrt{b^{2}+c^{2}}=\sqrt{\frac{a^{2}}{2} \mathbf{D}: \mathbf{D}+\frac{\chi^{2}}{4}\|\mathbf{H}\|^{4}+a \chi \mathbf{D}: \mathbf{H H}} \\
& =\sqrt{\frac{1}{2}\left[a \mathbf{D}+\chi \mathcal{H}_{0}\right]:\left[a \mathbf{D}+\chi \mathcal{H}_{0}\right]} .
\end{aligned}
$$

Here,

$$
\mathcal{H}_{0}=\mathbf{H H}-\frac{\|\mathbf{H}\|^{2}}{2}\left(\mathbf{e}_{1} \mathbf{e}_{1}+\mathbf{e}_{2} \mathbf{e}_{2}\right)
$$

and $a \mathbf{D}+\chi \mathcal{H}_{0}$ is the effective rate-of-strain tensor, which has two eigenvalues: $\pm \lambda$.

In the new configurational coordinates $\mathbf{n}=\mathbf{U} \cdot \mathbf{m}$, the Smoluchowski equation takes the form

$$
\begin{aligned}
& \frac{d \tilde{f}}{d t}= \mathcal{R}_{n} \cdot\left[D_{r}(a) \tilde{f} \mathcal{R}_{n} \tilde{\mu}\right] \\
&-\mathcal{R}_{n} \cdot\left[\mathbf{n} \times\left(\dot{\mathbf{n}}-\frac{\chi H^{2}}{2} \mathbf{e}_{3} \mathbf{e}_{3} \cdot \mathbf{n}\right) \tilde{f}\right], \\
& \dot{\mathbf{n}}=\tilde{\mathbf{W}} \cdot \mathbf{n}+\widetilde{a}[\tilde{\mathbf{D}} \cdot \mathbf{n}-\tilde{\mathbf{D}}: \mathbf{n n n}],
\end{aligned}
$$

where $\tilde{\mathbf{W}}=q \mathbf{W}_{0}, \widetilde{\mathbf{D}}=q \mathbf{D}_{0}$ are the effective vorticity and rate of strain tensor, respectively, and $\tilde{a}=\lambda / q$ is the modified geometry or shape parameter. ${ }^{18}$

This transformed system (13), by comparison with (3), corresponds to a simple shear flow with effective shear rate $2 q$, a modified shape parameter $\widetilde{a}$, together with an imposed magnetic field in the direction $\mathbf{e}_{3}$ normal to the shearing plane, and importantly, the anisotropy $-\chi / 2$ is opposite to the original one for the same material. The response to any coupled coplanar rotational flow and magnetic field is now given in terms of the solution of the target kinetic model (13), which we provide by a shear flow code extended to incorporate a transverse magnetic field component.

When $q=0$, the flow is irrotational; i.e., the vorticity tensor is zero. Here, we can choose

$$
\cos 2 \theta=\frac{c}{\sqrt{b^{2}+c^{2}}}, \quad \sin 2 \theta=\frac{b}{\sqrt{b^{2}+c^{2}}},
$$

so that the effective rate of strain tensor is transformed into a diagonal form $\lambda \mathbf{D}_{1}$, where

$$
\mathbf{D}_{1}=\left(\begin{array}{ccc}
1 & 0 & 0 \\
0 & -1 & 0 \\
0 & 0 & 0
\end{array}\right)
$$

Now, the corresponding flow in the reduced target model is a planar extension or elongation

$$
\mathbf{v}=\lambda(x,-y, 0),
$$

which is a potential flow with the corresponding normalized potential given by

$$
V_{e}=-\frac{\lambda}{2 D_{r}} \mathbf{D}_{1}: \mathbf{m m} \text {. }
$$

The problem can be written in terms of a scalar potential with the total normalized potential given by

$$
V=-\frac{3 N}{2} \mathbf{M}: \mathbf{m m}-\frac{\lambda}{2 D_{r}} \mathbf{D}_{1}: \mathbf{m m}+\frac{\chi}{4 D_{r}} H^{2} \mathbf{e}_{3} \mathbf{e}_{3}: \mathbf{m m},
$$

where the Maier-Saupe potential $V_{i}=-(3 N / 2) \mathbf{M}: \mathbf{m m}$ is adopted and $\mathbf{M}=\langle\mathbf{m m}\rangle$ is the second moment of $\mathbf{m}$ with respect to the pdf $f$. For this potential flow, the Smoluchowski equation is simplified to

$$
\frac{d}{d t} f=\mathcal{R} \cdot D_{r}(\mathcal{R} f+f \mathcal{R} V) .
$$

Thus, steady states are given by a Boltzmann distribution ${ }^{1}$

$$
f(\mathbf{m})=\frac{1}{Z} e^{-V}
$$

The proof of the above result can be found in Appendix B. In addition, in steady states, the ensemble averaged torque vanishes, i.e.,

$$
\langle\mathcal{R} V\rangle=0
$$

equivalent to

$$
\boldsymbol{\epsilon}_{i j k}\left(\frac{\lambda}{2 D_{r}} \mathbf{D}_{1}-\frac{\chi H^{2}}{4 D_{r}} \mathbf{e}_{3} \mathbf{e}_{3}\right)_{i l} \cdot \mathbf{M}_{l j}=0 .
$$

This in turn implies

$$
M_{12}=M_{13}=M_{23}=0 .
$$

Thus, the principal axes of the rate-of-strain tensor coincide with those of the second-moment tensor and both share the external field direction $\mathbf{e}_{3}$ as a principal axis.

The total potential can be recast into

$$
V=-\frac{3 N}{2} \mathbf{M}: \mathbf{m m}-\frac{1}{2} \mathbf{D}_{e x}: \mathbf{m m}+\text { const, }
$$

where

$$
\mathbf{D}_{\mathrm{ex}}=\frac{1}{D_{r}} \operatorname{diag}\left(\lambda+\frac{\chi H^{2}}{6},-\lambda+\frac{\chi H^{2}}{6},-\frac{\chi H^{2}}{3}\right)
$$

is an effective rate-of-strain tensor corresponding to a triaxial elongational flow with (generically) three unequal elongational or stretching rates. 
In the coordinate system with basis given by the three orthonormal eigenvectors of the second-moment tensor, the second moment is diagonal, with components given by

$$
\left\langle m_{i}^{2}\right\rangle=\int_{\|\mathbf{m}\|=1} m_{i}^{2} f(\mathbf{m}) d \mathbf{m}, \quad i=1,2,3 .
$$

We nondimensionalize the Smoluchowski equation by the time scale set by rotary diffusivity $t_{0}=1 / D_{r}$. The following dimensionless parameters then arise:

$$
\hat{p}=p t_{0}, \quad \hat{q}=q t_{0}, \quad \hat{\lambda}=\lambda t_{0}, \quad \hat{\chi}=\chi H_{0}^{2} t_{0}, \quad \hat{H}=\frac{1}{H_{0}} H
$$

where $H_{0}$ is a characteristic field strength. We identify $\mathrm{Pe}=2 \hat{q}$ as the Péclet number in simple shear or $\mathrm{Pe}=\hat{\lambda}$ as the effective Péclet number in simple planar elongation (extension). In the following, we will drop the hat ^ ${ }^{\wedge}$ on all dimensionless quantities.

\section{SIMPLE SHEAR FLOWS COUPLED WITH A TRANSVERSE MAGNETIC FIELD AND A NEGATIVE ANISOTROPY}

We now study parametric behavior of the reduced or target model for simple shear coupled to a transverse magnetic field of variable strength. We discretize the Smoluchowski equation using a spherical harmonic expansion: ${ }^{6,19-21}$

$$
f(\mathbf{m}, t)=\sum_{l=0}^{L} \sum_{m=-l}^{l} a_{l m}(t) Y_{l}^{m}(\mathbf{m}),
$$

where $Y_{l}^{m}$ are complex spherical harmonic functions, and $L$ is the order of truncation in the Galerkin approximation. After this discretization, the Smoluchowski equation is transformed to a system of ordinary differential equations for the coefficients $a_{l m}$. For the simulation results described below, we choose $L=10$, leading to a system of 65 coupled ordinary differential equations for the amplitudes. This choice gives robust results for small to moderate concentration $N$ and the strength of the anisotropy $\chi$.

Note that "in-plane" attractors, which are symmetric about the shearing plane, are characterized by the property that $a_{l m}=0$ for all odd integers $m ;^{6}$ otherwise, the attractor is out-of-plane. We utilize the continuation software AUTO $^{22}$ to produce all bifurcation diagrams presented below.

As we will see in the simulations, the impact of the transverse magnetic field for materials with a negative anisotropy is to assert an attraction toward the flow plane, or equivalently, a repulsion of the principal axis of the secondmoment tensor $\mathbf{M}$ away from the vorticity axis. One anticipates any steady or transient attractor to have attenuated outof-plane components compared to their zero magnetic field strength values. We will address the impact of the transverse magnetic field with respect to several representative regimes of the effective Péclet number. In the following discussion, we investigate how the variation in anisotropy and field strength $\widetilde{\mathbf{H}}$ affect the dynamics of the system at fixed values of $(q, \widetilde{a})$, where

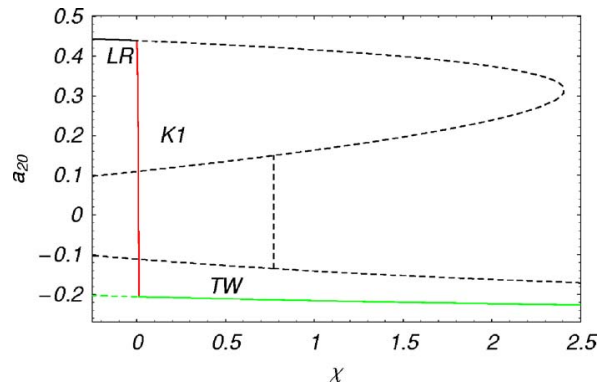

FIG. 1. Bifurcation diagram of $a_{2}^{0}$, the coefficient of the spherical harmonic mode $Y_{2}^{0}$ (time averaged for periodic states), as a function of anisotropy $\chi$ at parameter values $\mathrm{Pe}=1, \widetilde{a}=1$, and $N=5.5$. As $\chi$ increases, the logrolling (LR) steady states undergo a sharp unsteady transition to kayaking states $\left(K_{1}\right)$ and then collapse onto in-plane tumbling/wagging (TW) orbits. The solid curves indicate stability and other curves are unstable.

$$
\tilde{a}=\frac{1}{q} \sqrt{\left[\frac{\chi}{2}\left(H_{1}^{2}-H_{2}^{2}\right)\right]^{2}+\left(a p+\chi H_{1} H_{2}\right)^{2}} .
$$

This is a parametric study of the reduced model, in which we take liberty to decouple $\widetilde{a}$ and $\widetilde{\mathbf{H}}$ to present solution features of the Smoluchowski equation when flows and magnetic fields are strongly coupled. Note that in the study below, when we vary $\chi$ while freezing all other parameters in the reduced model, this does not correspond to an actual physical experiment. Nonetheless, for each target model parameter set, there is a corresponding three-parameter family of experiments with that behavior up to a transformation of the coordinate system and thereby the pdf.

As a backdrop for the simulations presented below, we recall from the phase diagram of sheared nematic polymers for high aspect ratio $(a \sim 1)$, and a dimensionless nematic concentration $(N=5.5$ and $N=5.1$ are used for illustrative purposes). ${ }^{7}$ This can be considered as an experiment in which one preshears a rigid rod suspension to generate an attractor. The results from the Doi-Hess kinetic theory are, as $\mathrm{Pe}$ is varied for these two nematic concentrations, nine distinct pdf attractor regions are resonated. We will then explore the consequences of turning on a transverse magnetic field with anisotropy parameter $\chi$, and study the magnetic fieldinduced transitions on each of these nine sheared attractors. The precise conditions that yield these sheared responses are: $\tilde{a}=1$ and $N=5.5$ for Figs. 1-6, with Péclet number $\mathrm{Pe}=2 q$

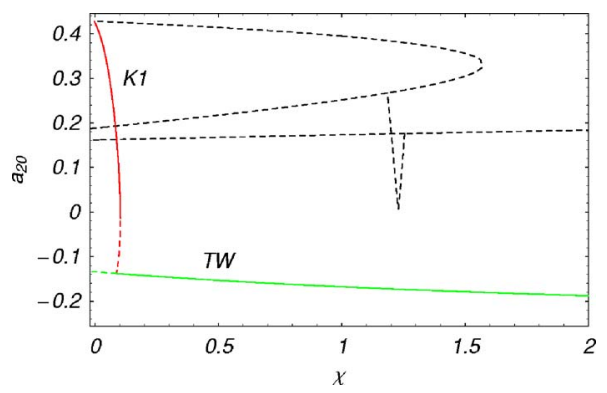

FIG. 2. Bifurcation diagram of $a_{2}^{0}$ as a function of anisotropy $\chi$ at parameter values $\mathrm{Pe}=3, \widetilde{a}=1$, and $N=5.5$. The shear response is $K_{1}$ for zero anisotropy $\chi=0$. As $\chi$ increases, the $K_{1}$ attractor collapses to a tumbling/wagging orbit (TW). In a very narrow band of $\chi$, the $K_{1}$ and TW attractors coexist. 


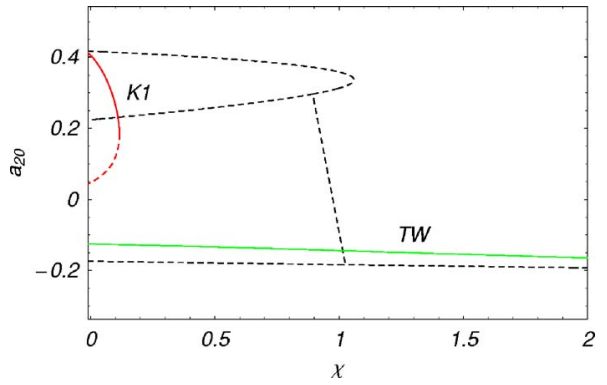

FIG. 3. Bifurcation diagram of $a_{2}^{0}$ as a function of anisotropy $\chi$ at parameter values $\mathrm{Pe}=4, \widetilde{a}=1$, and $N=5.5$. In this parameter regime, the bi-stable attractors are $K_{1}$ orbits in pure shear $(\chi=0)$ and the tumbling/wagging orbit (TW). As $\chi$ increases, the out-of-plane $K_{1}$ orbits disappear, leaving only the in-plane, tumbling-wagging orbit.

selected inside one of six intervals; $\widetilde{a}=1, N=5.1$ for Fig. 7, with two values of $\mathrm{Pe}=2 q$ that yield chaotic monodomain attractors, one with a bi-stable kayaking limit cycle; and then $\tilde{a}=1$ and $N=6$ for Fig. 8, with two values of Pe that yield tumbling/wagging attractors, one with a bistable logrolling steady state. In Ref. 23 , the role of the geometry parameter $\tilde{a}$ is extended beyond what is intended geometrically for the underlying molecules. Simulations were conducted for $\tilde{a}>1$, in which we show that the attractors are arrested by the unique flow-aligning steady state at a critical geometry parameter that depends on the nematic polymer concentration and the Péclet number when the magnetic field is absent. While the transverse magnetic field is present, our numerical calculations show that the same scenario remains (illustrated in Fig. 10). Finally, Figs. 11 and 12 report predictions for triaxial extensional flows, again at two distinct concentrations.

\section{A. Magnetic field-induced transitions of sheared logrolling steady states (Fig. 1)}

The stable attractor for $\mathrm{Pe}=1.5, \tilde{a}=1, N=5.5$, and $\chi=0$ is a so-called logrolling state (LR). This means the principal axis of the sheared pdf is aligned with the vorticity axis, and the solution is steady. When we turn on the magnetic field and increase its strength with negative anisotropy $-\chi$, the steady LR state persists only for very weak field strength, at

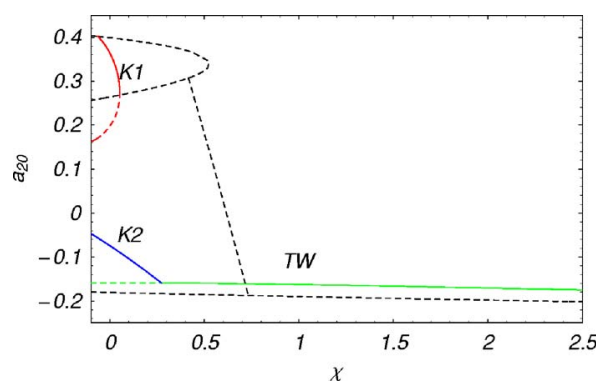

FIG. 4. Bifurcation diagram of $a_{2}^{0}$ as a function of anisotropy $\chi$ at parameter values $\mathrm{Pe}=5, \widetilde{a}=1$, and $N=5.5$. In this parameter regime, two stable attractors $\left(K_{1}\right.$ and $\left.K_{2}\right)$ coexist at small $\chi . K_{1}$ disappears after a turning point, leaving $K_{2}$ as a single attractor for a short interval of $\chi$. The out-of-plane tilted kayaking orbit $K_{2}$ then transitions into an in-plane tumbling/wagging orbit (TW) as $\chi$ increases.

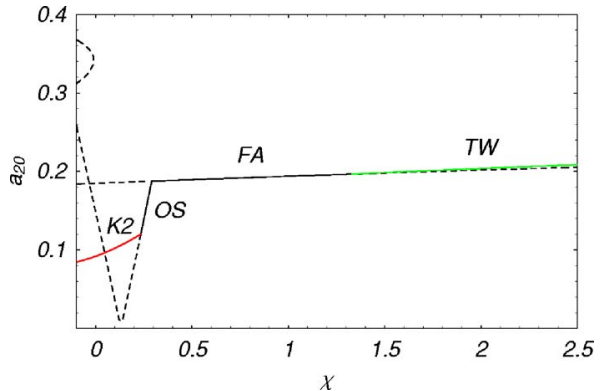

FIG. 5. Bifurcation diagram of $a_{2}^{0}$ as a function of anisotropy $\chi$ at parameter values $\mathrm{Pe}=6, \widetilde{a}=1$, and $N=5.5$. In this parameter regime, the stable attractor at small $\chi$ is $K_{2}$. It connects to a stable out-of-plane (OS) orbit as $\chi$ increases; the OS orbit comes down to a flow-aligning state as $\chi$ increases further. A tumbling/wagging orbit emerges at large values of $\chi$ !

which it undergoes a Hopf bifurcation to a kayaking limit cycle (with peak axis of the pdf rotating around the vorticity axis), which again only persists for a very limited range of weak magnetic field strength. Above a very weak field strength, the rod ensemble stabilizes in an in-plane tumbling limit cycle (the peak axis of the pdf rotates continuously in the flow-flow gradient plane, transverse to the magnetic field).

Thus, the magnetic field forces vorticity-aligned sheared steady states to in-plane periodic orbits. This is not a transition scenario that one might have predicted a priori on intuitive grounds. This phenomenon is furthermore not captured by the closure models that do not yield logrolling stable states, as do the Doi quadratic closure and its modifications. ${ }^{2}$

\section{B. Magnetic field-induced transitions of sheared kayaking limit cycles (Fig. 2)}

We raise the shear rate to $\mathrm{Pe}=3$, holding $\tilde{a}=1$ and $N=5.5$, with stable and unstable solutions of the reduced Smoluchowski equation versus magnetic field strength shown in Fig. 2. The pure shear response is a kayaking $\left(K_{1}\right)$ limit cycle at zero anisotropy $\chi=0$. As $\chi$ increases, the $K_{1}$ attractor "collapses" to a tumbling/wagging orbit (TW) in a hysteresis bifurcation. Bi-stable $K_{1}$ and tumbling/wagging solutions coexist in a very narrow band of $\chi$. This is once again a rather nonintuitive response diagram. However, once
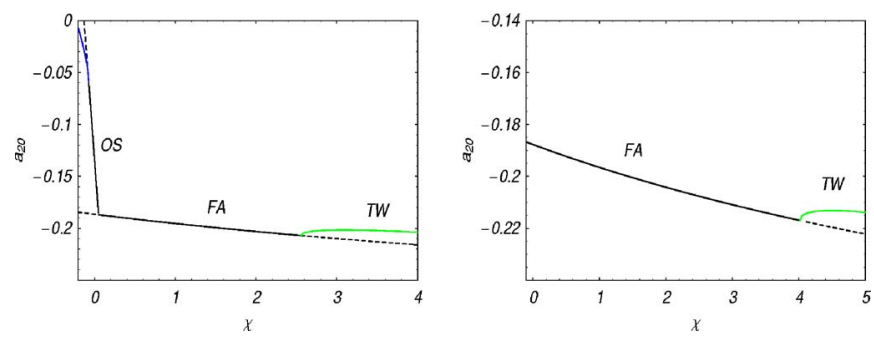

FIG. 6. Bifurcation diagram of $a_{2}^{0}$ as a function of anisotropy $\chi$ at parameter values $\tilde{a}=1, N=5.5$, and $\mathrm{Pe}=6.5$ (left), $\mathrm{Pe}=7$ (right). For $\mathrm{Pe}=6.5$, the stable sheared attractor absent of a magnetic field is a pair of out-of-plane stable states (OS). They collapse onto a flow-aligning steady state as $\chi$ increases, then a tumbling/wagging limit cycle. For $\mathrm{Pe}=7$, the stable attractor absent of magnetic field is a steady flow-aligned state (FA); then a tumbling/wagging orbit emerges at larger values of $\chi$. 

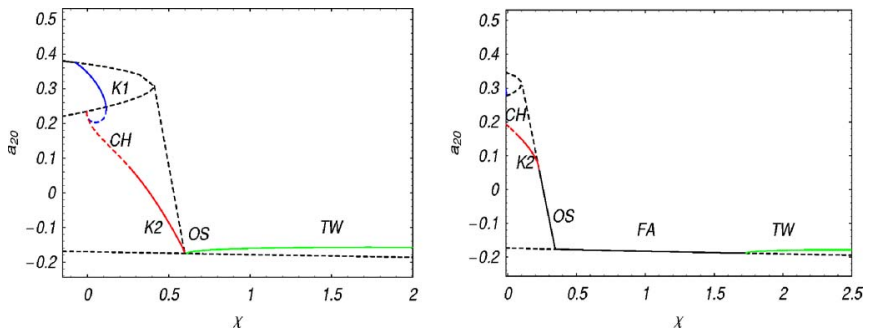

FIG. 7. Bifurcation diagram of $a_{2}^{0}$ as a function of anisotropy $\chi$ at parameter values $\tilde{a}=1, N=5.1$, and $\mathrm{Pe}=3$ (left), $\mathrm{Pe}=3.6$ (right). For $\mathrm{Pe}=3$ (left), the stable attractors absent of a magnetic field are $K 1$ and $\mathrm{CH}$. As $\chi$ increases, the $K 1$ attractor disappears, leaving only the chaotic state. Through period halving, the chaotic attractor then transitions to a pair of $K 2$, then steady out-of-plane states (OS), then in-plane steady states (FA), and finally TW limit cycles at sufficiently high amplitude. For $\mathrm{Pe}=3.6$ (right), the bifurcation sequence is similar.

again the magnetic field induces a transition to an in-plane limit cycle from an out-of-plane pure shear-induced response.

\section{Magnetic field-induced transitions of sheared bi-stable kayaking and tumbling/wagging limit cycles} (Fig. 3)

When $\mathrm{Pe}=4, \widetilde{a}=1, N=5.5$, and $\chi=0$, there are bistable $K_{1}$ and tumbling/wagging orbits. The tumbling/ wagging limit cycle persists for all $\chi>0$, whereas the $K_{1}$ solution survives up to a weak threshold value of $\chi>0$ and then vanishes by a turning point bifurcation. Once again, above a small threshold value of $\chi$, the only attractor is an in-plane limit cycle.

\section{Magnetic field-induced transitions of sheared bi-stable kayaking and tilted kayaking limit cycles (Fig. 4)}

At $\mathrm{Pe}=5, \tilde{a}=1$, and $N=5.5$, the sheared attractor set is a Larson-Ottinger kayaking limit cycle $K_{1}$ and a pair of tilted limit cycles $\left(K_{2}\right.$, whose pdf principal axes rotate around a direction between the vorticity axis and shear plane). As $\chi$ is turned on, the $K_{1}$ branch behaves qualitatively the same as the $\mathrm{Pe}=4$ scenario, vanishing at very low field strength. The $K_{2}$ solutions persist for somewhat higher field strength, then
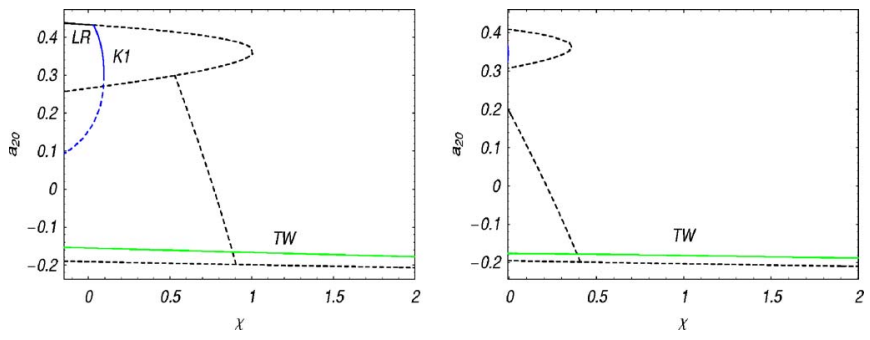

FIG. 8. Bifurcation diagram of $a_{2}^{0}$ as a function of anisotropy $\chi$ at parameter values $\tilde{a}=1, N=6$, and $\mathrm{Pe}=7$ (left), $\mathrm{Pe}=8.2$ (right). For $\mathrm{Pe}=7$ (left), the stable attractors absent of a magnetic field are LR and TW. As $\chi$ increases, the LR transitions to $K 1$ limit cycles, which disappear at a turning point, leaving only the TW limit cycle branch. For Pe $=8.2$ (right), the stable attractor absent of a magnetic field is the TW limit cycle, which persists as the magnetic field turns on and amplifies. are drawn down into the shear plane to a tumbling limit cycle, which at higher field strength merges into a wagging limit cycle. Again, although the pure shear attractor set is different from the above figures, above a critical $\chi$, the stable response is an in-plane limit cycle.

\section{E. Magnetic field-induced transitions of sheared tilted kayaking limit cycles (Fig. 5)}

At $\mathrm{Pe}=6, \tilde{a}=1, N=5.5$, and $\chi=0$, the sheared response is a pair of tilted kayaking orbits $K_{2}$. As the magnetic field is turned on and increased, a slightly different scenario unfolds than is observed at $\mathrm{Pe}=5$. First, there is a transition from the $K_{2}$ limit cycles to a pair of out-of-plane steady (OS) states, in a very small interval of $\chi$, which rapidly transition to inplane steady states (termed "FA" for flow-aligned, since the peak axis of the pdf is in the shear plane). Surprisingly, at higher $\chi$, the FA states transition to tumbling-wagging limit cycles. Again, we find that the starting sheared states are different, and the transition sequences for the lower ranges of magnetic anisotropy $\chi$ are also different, but above a threshold field strength, the attracting pdf is an in-plane limit cycle.

\section{F. Magnetic field-induced transitions of sheared out-of-plane and in-plane steady states (Fig. 6)}

For completeness, we shift to higher $\mathrm{Pe}=6.5$ and $\mathrm{Pe}=7$, for which the pure shear response is, respectively, an out-ofplane and in-plane, steady state. These steady attractors were already observed in the above transition diagrams, at finite nonzero values of $\chi$. We find that their transition behavior due to increased $\chi$ is the same here, where they are generated by pure shear first, and then a magnetic field is turned on and amplified. The steady in-plane alignment state (FA) occurs over a larger range of $\chi$, but above a threshold strength, an in-plane limit cycle is the attracting state.

\section{G. Magnetic field-induced transitions of sheared chaotic orbits (Fig. 7)}

Now we shift to lower concentration $N=5.1$, again with $\tilde{a}=1$, and first select $\mathrm{Pe}=3$ (left figure). This resonates a bi-stable attractor set with a kayaking orbit $K_{1}$ and a chaotic orbit $\mathrm{CH}$. As $\chi$ increases from zero, the $K_{1}$ attractor disappears, leaving only the chaotic state. A period halving cascade (the same sequence that is produced by increasing the shear rate $\left.{ }^{7,21}\right)$ ensues, and the chaotic attractor is arrested and replaced by a pair of tilted kayaking $K_{2}$ limit cycles. As seen in earlier figures, the $K_{2}$ limit cycles undergo a steady transition to OS steady states, which only exist for a very short range of $\chi$, and then tumbling/wagging limit cycles persist for higher $\chi$. For $\mathrm{Pe}=3.6$ (right figure), the chaotic attractor is unique for pure shear. The transition sequence versus $\chi$ is again a reverse period-halving arrest of the chaotic attractor to $K_{2}$ limit cycles, which undergo a transition to steady OS states, which now pass into in-plane steady FA states, and finally tumbling/wagging limit cycles above a threshold strength $\chi$. 

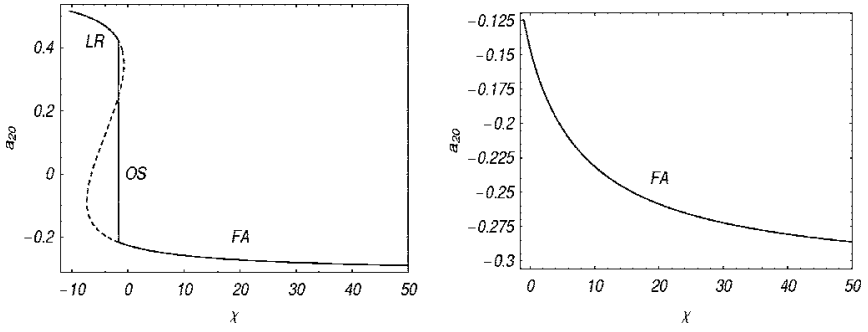

FIG. 9. Bifurcation diagram of $a_{2}^{0}$ as a function of anisotropy $\chi$ at parameter values $\widetilde{a}=1.15, N=5.5$, and $\mathrm{Pe}=6$ (left); $\widetilde{a}=1, N=4$, and $\mathrm{Pe}=6$ (right). In both cases, the stable attractors absent of a magnetic field are FA. As $\chi$ increases, the stable attractor remains FA. For $\widetilde{a}=1.15$, we plot the solution for $\tilde{a}<0$ (left), where a transition to LR via OS is shown as $\chi$ decreases.

\section{H. Magnetic field-induced transitions of high concentration, sheared logrolling, and tumbling orbits} (Fig. 8)

We raise the normalized concentration to $N=6$, again with $\tilde{a}=1$. For $\mathrm{Pe}=7$ (left), bi-stable attractors absent of a magnetic field are logrolling (LR) and tumbling/wagging (TW). As $\chi$ increases, the LR attractor transitions to $K 1$ limit cycles, which disappear again at a turning point bifurcation. The TW state persists for all $\chi$. For $\mathrm{Pe}=8.2$ (right), the stable attractor absent of a magnetic field is TW, which persists as the magnetic field is turned on and amplified.

\section{Magnetic field effects on in-plane flow-aligned stable steady states (Fig. 9)}

We recall that $\lambda$ is a measure of the effective rate of strain, while $q$ is a measure of the vorticity strength due to flows. The modified geometry parameter $\widetilde{a}=\lambda / q$ is thus a measure of the strain rate relative to the vorticity intensity, which includes the contributions from, molecular geometry, flow, material anisotropic property, and the magnetic field. As the magnetic field and flow field vary, this parameter may exceed 1 , and of course, it may also be reduced toward 0 . In our study on shear induced orientation responses, ${ }^{23}$ we showed that the limit cycles and other out-of-plane attractors as $\tilde{a}$ increases beyond 1.15 tend to be arrested by the flowaligned steady state. From the above interpretation of $\widetilde{a}$, this indicates that there exists a threshold value for the rate of stretching relative to the rotation of the flow field, beyond which the stable state is the flow-aligned attractor. Once a flow-aligned state is reached at fixed $\widetilde{a}>1$, increasing the magnetic field can only increase $|\widetilde{a}|$ and $\chi$, which will not disrupt the stability of the flow-aligned state nor resonate any limit cycles or other attractors. Figure 9 depicts a calculation that allows $\chi$ to vary from a negative value to a fairly large positive one at $\widetilde{a}=1.15$.

The system at smaller values of $N$ also normally admits one flow-aligned stable steady state. Turning on the magnetic field at fixed $\tilde{a}$ does not change the scenario qualitatively at all. Figure 9 also plots a representative case at $N=4$, where the only stable steady state is flow-aligned.

The target model at $0<\widetilde{a}<1$ and $\chi=0$ does not create any new types of attractors or limit cycles. Therefore, the orientational response to the increase of $\chi$ alluded to earlier remains qualitatively. The details may differ depending on how far $\tilde{a}$ is away from 1 , however.

This set of numerical experiments predicts that a magnetic field with negative anisotropy, imposed perpendicular to the flow-flow gradient plane in pure shear, yields two general phenomena: (i) the pdf is driven to in-plane distributions at a relatively low strength of the magnetic field and (ii) above a threshold field strength, independent of the pure sheared attractor (steady or unsteady, regular or chaotic), the pdf attractor can be an in-plane flow-aligned or tumbling/ wagging limit cycle. This nonlinear effect of magnetic anisotropy coupled to pure shear is a remarkable manifestation of rod ensemble response to coupled external flow and magnetic fields and nonlocal excluded volume interactions. Experimental verification would be helpful to validate the prediction.

\section{PLANAR EXTENSIONAL FLOWS COUPLED WITH A COPLANAR MAGNETIC FIELD}

In dimensionless variables, the steady state solutions of the Smoluchowski equation are governed by the Boltzmann distribution with potential (24), where

$$
\begin{aligned}
& V=-\frac{3 N}{2} \mathbf{M}: \mathbf{m m}-\frac{1}{2} D_{e}: \mathbf{m m}, \\
& D_{e}=\operatorname{diag}\left(\mathrm{Pe}+\frac{\chi}{6},-\mathrm{Pe}+\frac{\chi}{6},-\frac{\chi}{3}\right) .
\end{aligned}
$$

We choose coordinates aligned with the three principal axes of the second-moment tensor $\mathbf{M}, \mathbf{n}_{1,2,3}$, where $\mathbf{n}_{3}$ is the transverse external field direction taken as $\mathbf{e}_{3}$ in this paper. The orientation of the two in-plane directors $\mathbf{n}_{1}$ and $\mathbf{n}_{2}$ is determined by the "Leslie angle" $\theta$ calculated via (14). We parameterize the unit vector $\mathbf{m}$ by

$$
\mathbf{m}=\cos \tilde{\theta} \mathbf{n}_{1}+\sin \tilde{\theta} \cos \phi \mathbf{n}_{2}+\sin \tilde{\theta} \sin \phi \mathbf{n}_{3} .
$$

The second-moment tensor also admits a biaxial representation

$$
\mathbf{M}=s\left(\mathbf{n}_{1} \mathbf{n}_{1}-\frac{\mathbf{I}}{3}\right)+\beta\left(\mathbf{n}_{2} \mathbf{n}_{2}-\frac{\mathbf{I}}{3}\right),
$$

where $s$ and $\beta$ are the two order parameters that describe degrees of anisotropy of the pdf. If either $s$ or $\beta$ vanishes, or $s=\beta$, the pdf is uniaxial; otherwise, the pdf is fully biaxial. In the above parametrization, $s$ and $\beta$ satisfy the following equations:

$$
\begin{aligned}
& s-\frac{\beta}{2}=\left\langle\frac{3}{2} \cos ^{2} \tilde{\theta}-\frac{1}{2}\right\rangle, \\
& \beta=\left\langle\sin ^{2} \tilde{\theta} \cos 2 \phi\right\rangle,
\end{aligned}
$$

where

$$
\langle(\bullet)\rangle=\int_{\|\mathbf{m}\|=1}(\bullet) f d \mathbf{m},
$$



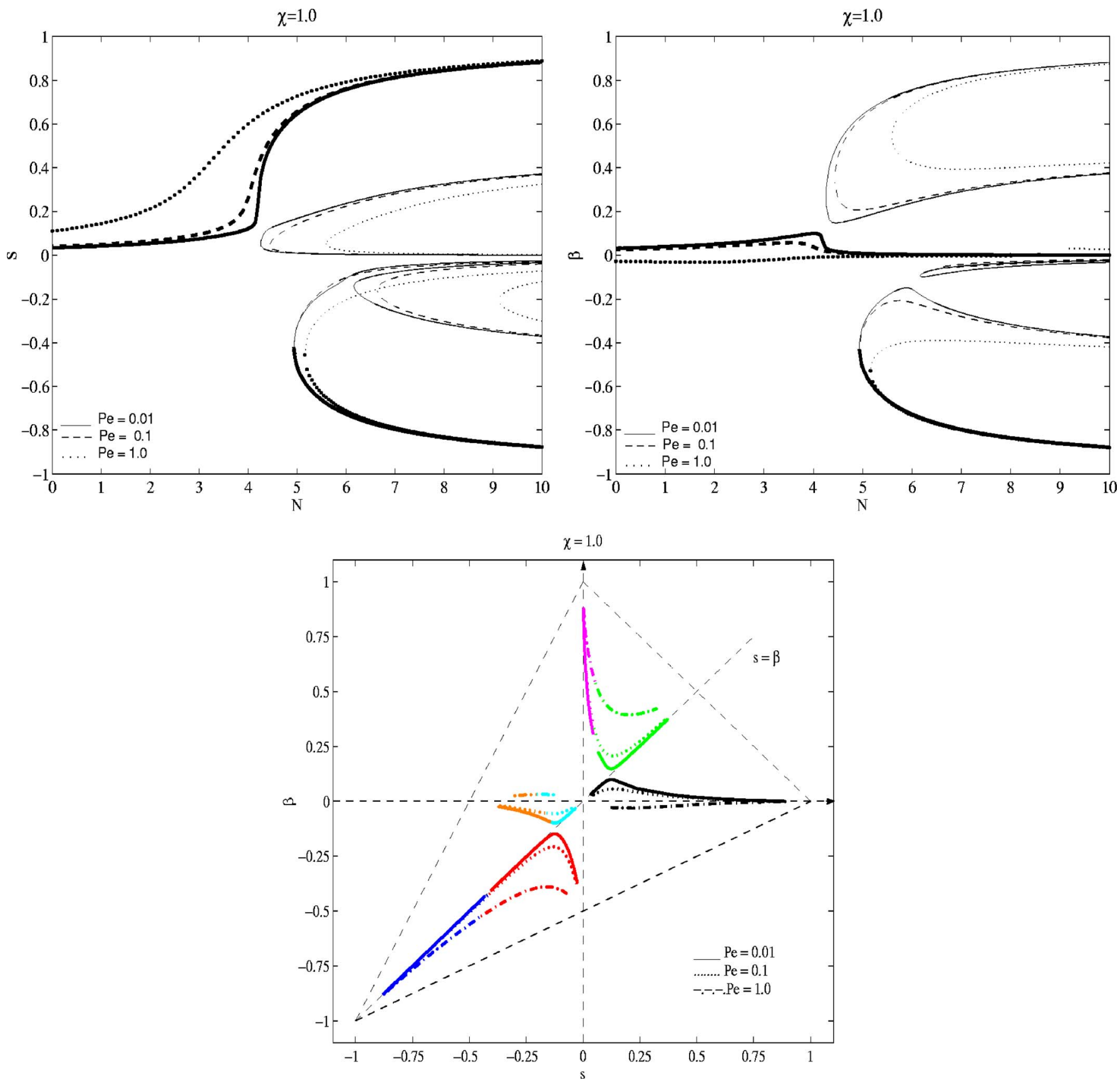

FIG. 10. Order parameter $s$ and $\beta$ as functions of $N$ at selected Péclet number $\mathrm{Pe}=0.01,0.1,1$. The anisotropy parameter is set at $\chi=1$. The flow-aligned equilibria (with peak axis in the plane of the flow) and the equilibria with peak axis perpendicular to the flow, are bi-stable. The flow-aligned state is the minimum energy equilibrium, in all cases. The solid black curves represent the stable branches.

$$
\begin{aligned}
f= & \frac{1}{Z} e^{h}, \\
h= & \frac{3 N}{2}\left[(s-\beta / 2) \cos ^{2} \tilde{\theta}+\frac{\beta}{2} \sin ^{2} \tilde{\theta} \cos 2 \phi\right] \\
& +\frac{1}{2}\left[\frac{3}{2}\left(\mathrm{Pe}+\frac{\chi}{6}\right) \cos ^{2} \tilde{\theta}+\left(\frac{-1}{2} \mathrm{Pe}+\frac{\chi}{4}\right) \sin ^{2} \tilde{\theta} \cos 2 \phi\right] .
\end{aligned}
$$

We first note that $\beta \neq 0$ in the steady states unless $(-\mathrm{Pe} / 2+\chi / 4)=0$. In fact, steady states are all biaxial if

$$
-\frac{1}{2} \mathrm{Pe}+\frac{\chi}{4} \neq 0
$$

Therefore, steady states are generically biaxial except in very special cases. We also note that for planar extension flows, we only need to discuss the case in which $\mathrm{Pe} \geq 0$ since $\mathrm{Pe}<0$ can be obtained through a $90^{\circ}$ planar rotation within the extension plane.

We denote the three non-negative eigenvalues of the second-moment tensor by $d_{1,2,3}$, associated with directors $\mathbf{n}_{1,2,3}$, respectively, which define the order parameters 

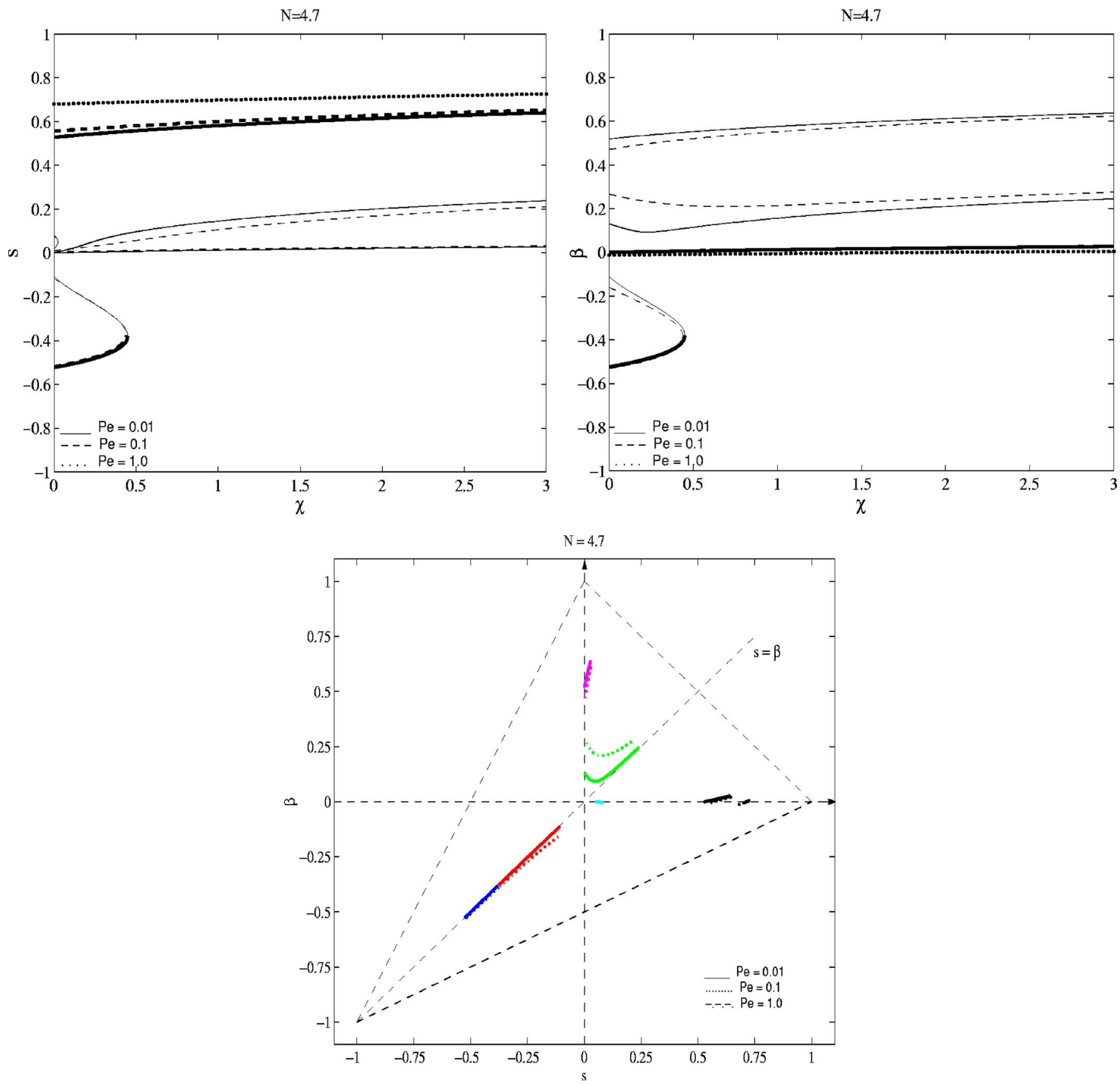

FIG. 11. Order parameters $s$ and $\beta$ as functions of $\chi$ at selected Péclet numbers $P e=0.01,0.1,1$ and concentration $N=4.7$. The flow-aligned and transversely aligned equilibria are the two stable branches, with the transverse alignment surviving only at small anisotropy. The solid black curves represent stable branches.

$$
s=d_{1}-d_{3}, \quad \beta=d_{2}-d_{3} .
$$

The only qualitative difference among equilibria is determined from the amplitudes and signs of $s$ and $\beta$, or equivalently, the relative order of $0 \leq d_{i} \leq 1$. Recall that the $\mathbf{e}_{3}$ axis associated with $d_{3}$ encodes the transverse magnetic field direction in the reduced model, so that either $d_{3}$ will be the largest eigenvalue, or not, in which case the principal axis is in the plane of the extensional flow. As we now illustrate, the principal axis shifts depending on the relative values of Pe and $\chi$, and there are typically multiple stable equilibria. However, the lowest energy equilibrium is always aligned in the plane of extensional flow, transverse to the magnetic field.

Figure 10 depicts the steady state solutions at three extension rates, $\mathrm{Pe}=0.01,0.1,1$, respectively, with fixed $\chi=1$. When $\mathrm{Pe}=0.01$, the effective rate-of-strain tensor is

$$
D_{e}=\operatorname{diag}(0.01+1 / 6,-0.01+1 / 6,-1 / 3) \text {. }
$$

All three directors align with the coordinate directions. The steady state with principal axis along the flow direction $\mathbf{e}_{1}$ is stable, with $s>0, \beta>0, s>\beta$, or equivalently, 

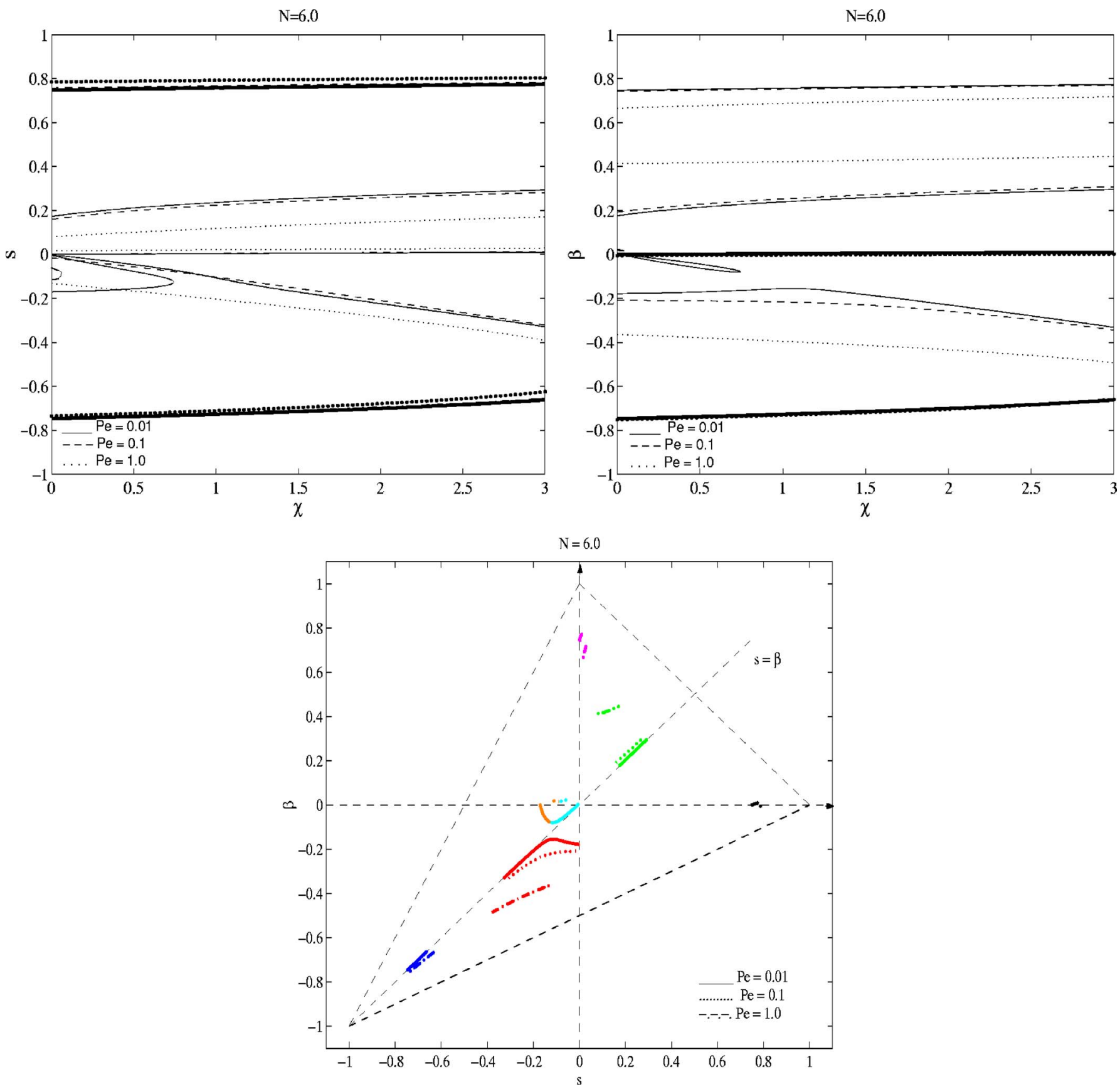

FIG. 12. Order parameters $s$ and $\beta$ as functions of $\chi$ at selected Péclet numbers $P e=0.01,0.1,1$ and concentration $N=4.7$. The flow-aligned and transversely aligned equilibria are the two stable branches. The solid black curves represent stable branches.

$$
d_{3}<d_{2}<d_{1} \text {. }
$$

(We infer stability by examining the second variation of the generalized free energy density; details are given in Appendix A.) This is completely expected, with all degrees of orientation $d_{i}$ correlated with the respective extension rates, $D_{e}^{i}$. The same flow plane aligned stable steady states persist out to $\mathrm{Pe}=0.1$. However, when $\mathrm{Pe}=1$, the extension rate $D_{e}^{3}$ along $\mathbf{e}_{3}$ exceeds $D_{e}^{2}$ along $\mathbf{e}_{2}$, and thus the $d_{i}$ follow the ordering

$$
d_{2}<d_{3}<d_{1} \text {. }
$$

Besides these stable steady states, there are other "metastable" states (linearly stable, but not a global minimum of the generalized free energy) given by a solution with the major director pointing in the direction perpendicular to the flow plane, for which $s<0, \beta<0$. One is tempted to call these logrolling, but they have nothing to do with shear flow! These steady equilibria are shown by the second family of solutions in Fig. 10, labeled "dark solid." We remark that this branch is unstable in most closure models. ${ }^{24-29}$

At fixed value of $\mathrm{Pe}$, the role of $\chi$ is to increase the extension rate in the extension plane while reducing it in the perpendicular direction. Intuitively, one anticipates that the degree of orientation will increase in the flow direction for flow-aligned equilibria, yet decrease in the transversely aligned steady state, and thus undergo an instability transi- 
tion at some critical magnetic field strength. This is indeed what happens. At concentrations less than $N=5$, the transversely aligned state only survives up to a finite value of $\chi$. However, when $N>5$, this branch persists for all $\chi$ in the range computed. Figures 11 and 12 depict all stable and unstable steady states at selected Péclet numbers for $N=4.7$ and $N=6$, respectively.

\section{CONCLUSION}

We have explored various phenomena associated with the strong coupling of coplanar linear flow and magnetic fields in rigid rod or platelet suspensions. The approach is based on a reciprocity relation of the Doi-Hess kinetic theory which reduces the problem to either of two simpler kinetic models, depending on whether the linear planar flow is rotational or irrotational. For rotational flows, we solve the kinetic equation with a new spherical harmonic Galerkin method, coupled with the continuation software AUTO. Various predictions are made, indicating a regularizing influence of a coplanar magnetic field on each type of sheared monodomain attractor. Remarkably, a robust and quite general prediction emerges: every sheared attractor (steady and unsteady, regular and chaotic) is drawn by a coplanar magnetic field into a flow-aligned steady state or tumbling/wagging limit cycle, above a threshold field strength. The transition sequence from zero magnetic field strength to the supercritical flow-aligned or tumbling/wagging response differs for each sheared attractor, but not the eventual steady state or limit cycle response. For irrotational flows, analytical results are given first based on a Boltzmann distribution for the pdf, followed by numerical solution of the resulting steady state equilibrium equations. It turns out that bi-stable equilibria are typical, with one global minimum energy state whose principal axis is aligned in the flow plane, and another stable, higher energy state with principal axis transverse to the flow plane. The upshot in coupled extensional and magnetic fields is twofold: bi-stability, and a shift in the axis of primary alignment of the minimum energy state that is controlled by the magnetic field.

\section{ACKNOWLEDGMENTS}

The authors acknowledge financial support from AFOSR Grants No. FA9550-05-1-0025 and No. FA9550-06-1-0063, NSF Grants No. DMS 0604891, No. DMS 0604912, No. DMS 0605029, and No. DMS 0626180, NASA URETI BIMat Award No. NCC-1-02037, and the Army Research Office.

\section{APPENDIX A: THE STABILITY AND THE SECOND VARIATION OF THE FREE ENERGY DENSITY}

The stability of the equilibria in irrotational flows coupled with the coplanar magnetic field is studied by the second variation of the generalized free energy density. The free energy density for the material system subject to potential flows or external fields is given by

$$
A[f]=k T\left[\int_{\|\mathbf{m}\|=1}\left(f \ln f+\frac{1}{2} V_{i}[f] f+V_{e} f\right) d \mathbf{m}\right] .
$$

We denote $f_{e}$ the equilibrium pdf solution and $\delta f_{1}$ the perturbation of the equilibrium solution. The second variation of the free energy density is then given by

$$
\delta^{2} A\left[f_{e}\right]=\frac{\delta^{2}}{2} k T \int_{\|\mathbf{m}\|=1}\left(\frac{f_{1}^{2}}{f_{e}}+V_{i}\left[f_{1}\right] f_{1}\right) d \mathbf{m} .
$$

Let $f_{1}=f_{e} f_{2}$. The second variation reduces to

$$
\delta^{2} A=\frac{\delta^{2}}{2} k T\left\langle f_{2}^{2}+V_{i}\left[f_{e} f_{2}\right] f_{2}\right\rangle_{e}
$$

where $\langle(\bullet)\rangle_{e}$ denotes the ensemble average taken with respect to the equilibrium pdf $f_{e}$. We introduce

$$
x=\frac{3 N}{2} \mathbf{M}: \mathbf{m m}
$$

where $\mathbf{M}$ is a symmetric matrix of trace zero. We consider the perturbation

$$
\begin{aligned}
& f_{2}=x-\langle x\rangle . \\
& \int_{\|\mathbf{m}\|=1} f_{2} d \mathbf{m}=0 .
\end{aligned}
$$

Here, we focus on a special perturbation that perturbs only the order parameters:

$$
x=\left[\widetilde{s}\left(m_{1}^{2}-1 / 3\right)+\widetilde{\beta}\left(m_{2}^{2}-1 / 3\right)\right]
$$

The equilibrium pdf is

$$
f=\frac{1}{Z} e^{(N / 2)\left[s\left(m_{1}^{2}-1 / 3\right)+\beta\left(m_{2}^{2}-1 / 3\right)\right]-V_{e}} .
$$

Derive the Hessian for the quadratic term with respect to $\tilde{s}, \tilde{\beta}$. We note that

$$
s=\left\langle m_{1}^{2}-m_{3}^{2}\right\rangle, \quad \beta=\left\langle m_{2}^{2}-m_{3}^{2}\right\rangle .
$$

In terms of the angles,

$$
s=\left\langle\cos ^{2} \theta-\sin ^{2} \theta \sin \phi\right\rangle, \quad \beta=\left\langle\sin ^{2} \theta \cos 2 \phi\right\rangle .
$$

The Hessian of the free energy density is given by 


$$
\begin{aligned}
\frac{\partial^{2} A}{\partial s^{2}}= & \left(\frac{3 N}{2}\right)^{2}\left[\left\langle m_{1}^{4}\right\rangle-\frac{2}{3}\left\langle m_{1}^{2}\right\rangle+\frac{1}{9}\right]-\left(\frac{3 N}{2}\right)^{3}\left[\left(\left\langle m_{1}^{4}\right\rangle-\frac{1}{3}\left\langle m_{1}^{2}\right\rangle\right)^{2}+\left(\left\langle m_{1}^{2} m_{2}^{2}\right\rangle-\frac{1}{3}\left\langle m_{2}^{2}\right\rangle\right)^{2}+\left(\left\langle m_{1}^{2} m_{3}^{2}\right\rangle-\frac{1}{3}\left\langle m_{3}^{2}\right\rangle\right)^{2}\right. \\
& \left.+2\left(\left\langle m_{1}^{3} m_{2}\right\rangle-\frac{1}{3}\left\langle m_{1} m_{2}\right\rangle\right)^{2}+2\left(\left\langle m_{1}^{3} m_{3}\right\rangle-\frac{1}{3}\left\langle m_{1} m_{3}\right\rangle\right)^{2}+2\left(\left\langle m_{1}^{2} m_{2} m_{3}\right\rangle-\frac{1}{3}\left\langle m_{2} m_{3}\right\rangle\right)^{2}\right] \frac{\partial^{2} A}{\partial \beta^{2}} \\
= & \left(\frac{3 N}{2}\right)^{2}\left[\left\langle m_{2}^{4}\right\rangle-\frac{2}{3}\left\langle m_{2}^{2}\right\rangle+\frac{1}{9}\right]-\left(\frac{3 N}{2}\right)^{3}\left[\left(\left\langle m_{2}^{4}\right\rangle-\frac{1}{3}\left\langle m_{2}^{2}\right\rangle\right)^{2}+\left(\left\langle m_{1}^{2} m_{2}^{2}\right\rangle-\frac{1}{3}\left\langle m_{1}^{2}\right\rangle\right)^{2}+\left(\left\langle m_{2}^{2} m_{3}^{2}\right\rangle-\frac{1}{3}\left\langle m_{3}^{2}\right\rangle\right)^{2}\right. \\
& \left.+2\left(\left\langle m_{2}^{3} m_{1}\right\rangle-\frac{1}{3}\left\langle m_{1} m_{2}\right\rangle\right)^{2}+2\left(\left\langle m_{2}^{3} m_{3}\right\rangle-\frac{1}{3}\left\langle m_{2} m_{3}\right\rangle\right)^{2}+2\left(\left\langle m_{2}^{2} m_{1} m_{3}\right\rangle-\frac{1}{3}\left\langle m_{1} m_{3}\right\rangle\right)^{2}\right] \frac{\partial^{2} A}{\partial s \partial} \\
= & \left(\frac{3 N}{2}\right)^{2}\left[\left\langle m_{1}^{2} m_{2}^{2}\right\rangle-\frac{1}{3}\left\langle m_{1}^{2}\right\rangle-\frac{1}{3}\left\langle m_{2}^{2}\right\rangle+\frac{1}{9}\right]-\left(\frac{3 N}{2}\right)^{3}\left[\left(\left\langle m_{1}^{4}\right\rangle-\frac{1}{3}\left\langle m_{1}^{2}\right\rangle\right)\left(\left\langle m_{1}^{2} m_{2}^{2}\right\rangle-\frac{1}{3}\left\langle m_{1}^{2}\right\rangle\right)\right. \\
& +\left(\left\langle m_{2}^{4}\right\rangle-\frac{1}{3}\left\langle m_{2}^{2}\right\rangle\right)\left(\left\langle m_{1}^{2} m_{2}^{2}\right\rangle-\frac{1}{3}\left\langle m_{2}^{2}\right\rangle\right)+\left(\left\langle m_{2}^{2} m_{3}^{2}\right\rangle-\frac{1}{3}\left\langle m_{3}^{2}\right\rangle\right)\left(\left\langle m_{1}^{2} m_{3}^{2}\right\rangle-\frac{1}{3}\left\langle m_{3}^{2}\right\rangle\right) \\
& +2\left(\left\langle m_{2}^{3} m_{1}\right\rangle-\frac{1}{3}\left\langle m_{1} m_{2}\right\rangle\right)\left(\left\langle m_{1}^{3} m_{2}\right\rangle-\frac{1}{3}\left\langle m_{1} m_{2}\right\rangle\right)+2\left(\left\langle m_{2}^{3} m_{3}\right\rangle-\frac{1}{3}\left\langle m_{2} m_{3}\right\rangle\right)\left(\left\langle m_{1}^{2} m_{2} m_{3}\right\rangle-\frac{1}{3}\left\langle m_{2} m_{3}\right\rangle\right) \\
& \left.+2\left(\left\langle m_{2}^{2} m_{1} m_{3}\right\rangle-\frac{1}{3}\left\langle m_{1} m_{3}\right\rangle\right)\left(\left\langle m_{1}^{3} m_{3}\right\rangle-\frac{1}{3}\left\langle m_{1} m_{3}\right\rangle\right)\right] .
\end{aligned}
$$

\section{APPENDIX B: PROOF OF THE EQUIVALENCE OF THE HYDRODYNAMIC EQUILIBRIUM AND THE THERMODYNAMIC EQUILIBRIUM}

Theorem. The steady state solution of the Smoluchowski equation is given by the Euler-Lagrange equation of the free energy density.

Proof. The free energy density for homogeneous flows of rigid nematic polymers or rod/platelet dispersions is given by

$$
A[f]=k T \int_{\|\mathbf{m}\|=1}\left[f \ln f+\frac{V_{i}}{2} f+V_{e} f\right] d \mathbf{m},
$$

where $V_{i}$ and $V_{e}$ are the intermolecular potential and the external potential, respectively. The Euler-Lagrange equation obtained from minimizing the free energy density functional is

$$
\mu-\lambda=0,
$$

where

$$
\mu=\frac{\delta A}{\delta f}=k T\left[\ln f+V_{i}+V_{e}\right]
$$

is the extended chemical potential and $\lambda$ is the Lagrange multiplier with respect to the constraint

$$
\int_{\|\mathbf{m}\|=1} f d \mathbf{m}=1
$$

$\mu=\lambda$ is the governing equation for the thermodynamic equilibria.

The steady state solution of the Smoluchowski equation is governed by

$$
\mathcal{R} \cdot(f \mathcal{R} \mu)=0 .
$$

We multiply the equation by $\mu$ and then integrate over the unit sphere $\|\mathbf{m}\|=1$ to yield

$$
\int_{\|\mathbf{m}\|=1} \mu \mathcal{R} \cdot f \mathbf{R} \mu d \mathbf{m}=-\int_{\|\mathbf{m}\|=1} f\|\mathcal{R} \mu\|^{2} d \mathbf{m}=0 .
$$

This implies

$$
f(\mathbf{m}, t) \mathcal{R} \mu=0 .
$$

Finally,

$$
\mathcal{R} \mu=0 \text {. }
$$

This is equivalent to $\mu=\lambda$.

${ }^{1}$ Q. Wang, S. Sircar, and H. Zhou, "Steady state solutions of the Smoluchowski equation for nematic polymers under imposed fields," Commun. Math. Sci. 4, 605 (2005).

${ }^{2}$ M. G. Forest, Q. Wang, R. Zhou, and E. Choate, "Monodomain response of arbitrary aspect ratio nematic polymers in general linear planar flows," J. Non-Newtonian Fluid Mech. 118, 17 (2004).

${ }^{3}$ A. S. Bhandar and J. M. Wiest, "Mesoscale constitutive modeling of magnetic dispersions," J. Colloid Interface Sci. 257, 371 (2003).

${ }^{4}$ M. Doi and S. F. Edwards, The Theory of Polymer Dynamics (Clarendon, Oxford, 1986).

${ }^{5}$ S. Hess, "Fokker-Planck-equation approach to flow alignment in liquid crystals," Z. Naturforsch. A 31A, 1034 (1976).

${ }^{6}$ M. G. Forest, Q. Wang, and R. Zhou, "The weak shear phase diagram for nematic polymers," Rheol. Acta 43, 17 (2004).

${ }^{7}$ M. G. Forest, Q. Wang, and R. Zhou, "The flow-phase diagram of DoiHess theory for sheared nematic polymers II: finite shear rates," Rheol. Acta 44, 80 (2004).

${ }^{8}$ P. Constantin, I. Kevrekidis, and E. S. Titi, "Asymptotic states of a Smoluchowski equation," Arch. Ration. Mech. Anal. 174, 365 (2004).

${ }^{9}$ P. Constantin, I. Kevrekidis, and E. S. Titi, "Remarks on a Smoluchowski equation,” Discrete Contin. Dyn. Syst. 11, 101 (2004).

${ }^{10} \mathrm{P}$. Constantin and J. Vukadinovic, "Note on the number of steady states for a 2D Smoluchowski equation," Nonlinearity 18, 441 (2005). 
${ }^{11}$ H. Liu, H. Zhang, and P. Zhang, "Axial symmetry and classification of stationary solutions of Doi-Onsager equation on the sphere with MaierSaupe potential," Commun. Math. Sci. 3, 201 (2005).

${ }^{12}$ C. Luo, H. Zhang, and P. Zhang, "The structure of equilibrium solutions of the one-dimensional Doi equation," Nonlinearity 18, 379 (2005).

${ }^{13}$ I. Fatkullin and V. Slastikov, "Critical points of the Onsager functional on a sphere," Nonlinearity 18, 2565 (2005).

${ }^{14}$ A. Isihara, "The theory of anisotropic colloidal solutions," J. Chem. Phys. 19, 1142 (1951).

${ }^{15}$ N. Kuzuu and M. Doi, "Constitutive equations for nematic liquid crystals under weak velocity gradients derived from a molecular kinetic equation," J. Phys. Soc. Jpn. 52, 3486 (1983).

${ }^{16} \mathrm{Q}$. Wang, "A hydrodynamic theory of nematic liquid crystalline polymers of different configurations," J. Chem. Phys. 116, 9120 (2002).

${ }^{17}$ P. G. deGennes and J. Prost, The Physics of Liquid Crystals (Oxford University Press, New York, 1993).

${ }^{18}$ M. G. Forest, R. Zhou, and Q. Wang, "Monodomain response of arbitrary aspect ratio nematic polymers in general linear planar flows," J. NonNewtonian Fluid Mech. 118, 17 (2004)

${ }^{19}$ R. G. Larson and H. C. Ottinger, "Effect of molecular elasticity on outof-plane orientations in shearing flows of liquid crystalline polymers," Macromolecules 24, 6270 (1991).

${ }^{20}$ V. Faraoni, M. Grosso, S. Crescitelli, and P. L. Maffettone, "The rigid-rod model for nematic polymers: An analysis of the shear flow problem," J. Rheol. 43, 829 (1999).

${ }^{21}$ M. Grosso, R. Keunings, S. Crescitelli, and P. L. Maffettone, "Prediction of chaotic dynamics in sheared liquid crystalline polymers," Phys. Rev. Lett. 86, 3184 (2001).

${ }^{22}$ E. J. Doedel, A. R. Champneys, T. F. Fairgrive, Y. A. Kuznetsov, B. Sandstede, and X. Wang, AUTO97: Continuation and Bifurcation Software for Ordinary Differential Equations (Concordia University, Montreal, Canada, 1997).

${ }^{23}$ M. G. Forest, R. Zhou, and Q. Wang, "Chaotic boundaries of nematic polymers in mixed shear and extensional flows," Phys. Rev. Lett. 93, 088301 (2004).

${ }^{24}$ A. Rey, "Bifurcational analysis of the isotropic-nematic phase transition of rigid rod polymers subjected to biaxial stretching flow," Macromol. Theory Simul. 4, 857 (1995).

${ }^{25}$ A. Rey, "Bifurcational analysis of the isotropic-discotic nematic phase transition in the presence of extensional flow," Liq. Cryst. 19, 325 (1995).

${ }^{26} \mathrm{Q}$. Wang, "Comparative studies on closure approximations in flows of liquid crystal polymers. I. Elongational flows," J. Non-Newtonian Fluid Mech. 72, 141 (1997).

${ }^{27}$ M. G. Forest, Q. Wang, and H. Zhou, "Homogeneous biaxial patterns and director instabilities of liquid crystal polymers in axial and planar elongation," Phys. Fluids 12, 490 (2000).

${ }^{28}$ M. G. Forest, Q. Wang, and H. Zhou, "On the flow-phase diagram for discotic liquid crystals in uniaxial extension and compression," Liq. Cryst. 5, 717 (2001).

${ }^{29}$ H. Zhou, H. Wang, Q. Wang, and M. G. Forest, "A new proof on uniaxial equilibria of a 3-dimensional Smoluchowski equation," Nonlinearity 18, 2815 (2005). 